\title{
DESLOCAMENTO VERTICAL DE NEMATÓIDES ENTOMOPATOGÊNICOS (Rhabditida: Heterorhabditidae) NA BUSCA POR Dysmicoccus texensis (Tinsley) (Hemiptera: Pseudococcidae) EM LABORATÓRIO E CASA-DE-VEGETAÇÃo
}

\author{
Vertical displacement of entomopathogenic nematodes (Rhabditida: Heterorhabditidae) \\ in search of Dysmicoccus texensis (Tinsley) (Hemiptera: Pseudococcidae) under laboratory \\ and greenhouse conditions
}

\author{
Viviane Sandra Alves ${ }^{1}$, Alcides Moino Junior ${ }^{2}$
}

\begin{abstract}
RESUMO
Vários fatores devem ser considerados na avaliação da eficiência de um entomopatógeno no controle de um inseto-praga. Com relação aos nematóides entomopatogênicos, além da patogenicidade e virulência, é importante conhecer a capacidade de busca, pois quanto maior sua eficiência, maior a chance de encontro com o hospedeiro. Assim, neste trabalho, objetivou-se avaliar o deslocamento vertical de nematóides entomopatogênicos (Heterorhabditis), visando ao controle da cochonilha-da-raiz-do-cafeeiro Dysmicoccus texensis (Tinsley). Para avaliação em laboratório, foi feito teste em coluna de areia de $5 \mathrm{~cm}$ (em delineamento inteiramente casualizado) onde os isolados CCA e JPM3 foram aplicados em suspensão aquosa no topo da coluna em três concentrações (50, 100 e $500 \mathrm{JIs} /$ inseto), com avaliação da mortalidade após 5 dias. No experimento de coluna de solo, foi usada uma coluna de $25 \mathrm{~cm}$, composta por cinco extratos de $5 \mathrm{~cm}$. Foram utilizados dois isolados (CCA e JPM3) e dois métodos de aplicação: cadáver infectado e suspensão aquosa, ambos avaliados nas diferentes profundidades. O experimento foi conduzido num delineamento fatorial $3 \times 2 \times 5$, em condições de casa-de-vegetação. No experimento de coluna de areia, não houve diferença entre os isolados avaliados e ambos alcançaram valor de $92 \%$ de mortalidade. No experimento de deslocamento em coluna de solo, observou-se que JPM3 foi mais eficiente que CCA, nos dois métodos de aplicação avaliados. Quanto aos métodos de aplicação, o de suspensão aquosa apresentou melhores resultados para os dois isolados, e JPM3 aplicado em suspensão aquosa, foi o melhor tratamento em quase todas as profundidades avaliadas.
\end{abstract}

Termos para indexação: Controle Microbiano, "cruiser", "ambusher", pragas do café.

\section{ABSTRACT}

Many factors must be considered in evaluating the efficiency of an entomopathogen in the control of an insect pest. With regards to entomopathogenic nematodes, in addition to their pathogenicity and virulence, it is important to know the ability to search the host, which is directly linked with its efficiency. The objective of this study was to evaluate the vertical displacement of entomopathogenic nematodes (Heterorhabditis) to control the coffee root scale Dysmicoccus texensis (Tinsley). A test was conducted in a sand column of $5 \mathrm{~cm}$ depth under laboratory conditions, using a randomized design, with the strains CCA and JPM3 being applied in aqueous suspension at the top of the column in three concentrations (50, 100 and $500 \mathrm{IJs} /$ insect), with evaluation of mortality after 5 days. It was also conducted an experiment in a $2 \times 2 \times 6$ factorial design, at greenhouse conditions with a soil column of $30 \mathrm{~cm}$ depth, composed of six extracts of $5 \mathrm{~cm}$. Two nematode strains (CCA and JPM3) and two methods of application (infected cadaver and aqueous suspension) were evaluated at different depths. There was no difference between the strains evaluated in the laboratory experiment, and both reached values of $92 \%$ mortality. In the experiment conducted at the greenhouse, it was observed that JPM3 was more efficient than CCA, at the two methods of application assessed. Regarding With application methods, aqueous suspension showed better results for both strains and JPM3 was the best treatment in almost all depths evaluated.

Index terms: Microbial Control, “cruiser", "ambusher", coffee pests.

(Recebido em 9 de abril de 2008 e aprovado em 23 de setembro de 2008)

\section{INTRODUÇÃO}

Os nematóides entomopatogênicos das famílias Steinernematidae e Heterorhabditidae têm se mostrado agentes promissores e alternativos ao controle químico de muitos insetos-praga e sua utilização em programas de Manejo Integrado de Pragas (MIP) já é realidade em muitos países (Kaya, 1985; Georgis et al., 2005).

Entre as vantagens apresentadas pelos nematóides entomopatogênicos, com relação a outros agentes de controle microbiano, está a capacidade de busca pelo hospedeiro. Nesse sentido, eles podem apresentar dois tipos de comportamento: "cruiser" e "ambusher". Esta classificação é baseada na diferença de tempo que o nematóide passa parado à espreita do hospedeiro ("ambusher") e no tempo em que ele se desloca em busca do hospedeiro ("cruiser") (Huey \& Pianka, 1981; Brien et al., 1989). Alguns nematóides podem apresentar comportamento intermediário.

${ }^{1}$ Graduada em Ciências Biológicas, Pós-doc - Departamento de Ciências Biológicas - Universidade Estadual do Norte Paranaense/UENP - Avenida Portugal, 340 - 86300-000 -Cornélio Procópio, PR - vivialves21@hotmail.com

'Engenheiro Agrônomo, Doutor em Entomologia - Departamento de Entomologia/DEN - Universidade Federal de Lavras/UFLA - Cx. P. 3037 - $37200-000$ Lavras, MG - alcmoino@ufla.br 
Nematóides com comportamento "cruiser" têm alta probabilidade de sucesso no controle de insetos-praga com hábito sedentário ou críptico, enquanto que nematóides com comportamento "ambusher" são mais indicados para o controle de insetos ativos, que se movimentam no ambiente (Lewis et al., 2006). Outro aspecto interessante, relacionado a essa capacidade de movimentação no perfil do solo, é a possibilidade de supressão competitiva de populações de nematóides fitopatogênicos. Segundo Salgado et al.(2007), Meloidogyne exigua Goeldi, 1887 é um nematóide que causa perdas severas na produção cafeeira, devido a alterações no estado nutricional do cafeeiro parasitado, decorrente da deficiente absorção e translocação de água e nutrientes, e sua redução populacional por meio de medidas ambientalmente aceitáveis deve ser considerada, assim como de outras espécies de nematóides e pragas em lavouras infestadas.

A cochonilha-da-raiz-do-cafeeiro, Dysmicoccus texensis (Tinsley) (Hemiptera: Pseudococcidae), é um exemplo de inseto com hábito críptico e seu ataque tem causado sérios prejuízos à cultura do cafeeiro (Souza \& Ribeiro, 2003). Os insetos instalam-se na raiz principal, na região abaixo do colo da planta, e distribuem-se para as raízes secundárias nas quais, associados ao fungo Bornetina sp., formam as chamadas criptas ou "pipocas", no interior das quais se alojam. As criptas impedem a absorção de água e nutrientes pela planta, que, em altas infestações, pode morrer (Nakano, 1972; Santa-Cecília et al., 2000; Sousa et al., 2001).

O hábito críptico desse inseto dificulta o controle químico e a ação de inimigos naturais como parasitóides e fungos entomopatogênicos. Por outro lado, os nematóides entomopatogênicos têm demonstrado um bom potencial para o controle desse inseto (Andaló et al., 2004a,b). Assim, conduziu-se este trabalho, com o objetivo de avaliar a capacidade de deslocamento vertical de dois isolados de nematóides entomopatogênicos (Heterorhabditis sp. CCA e JPM3), aplicados por meio de dois diferentes métodos, na busca de D. texensis, em condições de laboratório e casa-de-vegetação.

\section{MATERIAL E MÉTODOS}

\section{Criação de Dysmicoccus texensis}

A criação de $D$. texensis foi conduzida no laboratório de Patologia de Insetos da Universidade Federal de Lavras - UFLA, em Lavras - MG, no Campus da UFLA.

Como substrato para criação das cochonilhas, foram utilizadas abóboras do tipo moranga, variedade "Cabotcha". A infestação foi feita colocando pedaços de abóbora já infestados sobre abóboras novas, esperando pela passagem das ninfas e adultos da cochonilha. Assim que a infestação ocorreu, os pedaços foram retirados para evitar a contaminação com fungos. A criação foi mantida em condições controladas de temperatura de $27 \pm 1^{\circ} \mathrm{C}$, com umidade relativa de $70 \pm 10 \%$ e no escuro, em câmara climatizada a $27 \pm 1^{\circ} \mathrm{C}, 70 \pm 10 \%$ de umidade e no escuro.

\section{Obtenção de isolados de nematóides}

Os isolados utilizados foram Heterorhabditis sp. CCA (Araras, SP - Brasil) e Heterorhabditis sp. JPM3 (Lavras, MG - Brasil), obtidos do Banco de Entomopatógenos do laboratório de Patologia de Insetos da UFLA, sendo armazenados em frascos Erlenmeyer em suspensão aquosa, em condições controladas a $16 \pm 1^{\circ} \mathrm{C}$, com umidade relativa de $70 \pm 10 \%$, no escuro e com a concentração de até 500 juvenis infectivos (JIs)/mL.

Quando necessária, a multiplicação foi feita em larvas de último ínstar da traça-dos-favos, Galleria mellonella L. (Lepidoptera: Pyralidae), provenientes do laboratório de Biologia de Insetos do Departamento de Entomologia da UFLA.

\section{Deslocamento em coluna de areia (Teste em Laboratório)}

O teste de deslocamento em coluna de areia foi conduzido no laboratório de Patologia de Insetos do Departamento de Entomologia da UFLA. Foram usadas placas de Petri de $5 \mathrm{~cm}$ de diâmetro, com $20 \mathrm{~g}$ de areia grossa esterilizada + broto de batata para onde foram transferidas 10 fêmeas adultas da cochonilha. Em seguida, um cilindro de PVC de $5 \mathrm{~cm}$ de altura e $4 \mathrm{~cm}$ de diâmetro foi colocado sobre a base da placa, mantendo-se o broto no fundo, e então, o cilindro foi preenchido com areia até o topo (aproximadamente $80 \mathrm{~g}$ ). A areia foi umedecida com $8 \mathrm{~mL}$ de água destilada ( $10 \%$ do peso da areia) e, a seguir, as suspensões de nematóides foram aplicadas em três concentrações (50, 100 e $500 \mathrm{JIs} /$ inseto). O cilindro de PVC foi coberto com a tampa da placa de Petri, armazenado em caixas plásticas, contendo algodão umedecido e mantidas em câmara climatizada a $27 \pm 1^{\circ} \mathrm{C}, 70 \pm 10 \%$ de umidade e no escuro. Cada tratamento foi repetido cinco vezes. A avaliação foi feita após cinco dias, sendo os insetos mortos transferidos para câmara seca, para confirmação da mortalidade por meio da da observação da sintomatologia. Para o tratamento testemunha, foram seguidos os mesmos passos, porém esse tratamento recebeu aplicação apenas de água destilada esterilizada. O experimento foi conduzido em delineamento inteiramente casualizado e os dados de mortalidade foram submetidos à análise de variância e ao teste Scott-Knott $(\mathrm{P}<0,05)$ para comparação entre as médias.

\section{Deslocamento em coluna de solo (teste em casa-de- vegetação)}

Os dois isolados foram também avaliados quanto à capacidade de deslocamento em coluna de solo, simulando uma situação mais próxima às condições de campo. 
Para montagem da coluna foram utilizados cilindros de PVC de $150 \mathrm{~mm}$ de diâmetro, com altura de $5 \mathrm{~cm}$, com tela plástica colada em uma das extremidades. Cada cilindro, após ser preenchido com solo (Latossolo Vermelho Amarelo), recebeu um broto de batata sobre o qual foram transferidos 10 insetos. Os cilindros foram então empilhados até a altura de $25 \mathrm{~cm}$ (cinco cilindros) e unidos com fita adesiva.

Foram avaliados dois métodos de aplicação: suspensão aquosa $(150 \mathrm{~mL} /$ coluna $)$ e pelo método de cadáver infectado (larvas de G. mellonella, previamente infestadas), sendo usada uma larva por coluna, enterrada na superfície da mesma. A suspensão aquosa foi aplicada no topo da coluna na concentração de $28 \mathrm{JIs} / \mathrm{cm}^{2}$ para CCA e $29 \mathrm{JIs} / \mathrm{cm}^{2}$ para JPM3. Após a aplicação, as colunas de solo foram mantidas em casa-de-vegetação do Departamento de Entomologia da UFLA. A avaliação foi feita cinco dias após, desmontando-se as colunas e contando o número de insetos vivos e mortos em cada extrato da coluna.

$\mathrm{O}$ experimento foi conduzido em delineamento inteiramente casualizado, com esquema fatorial 3 (dois isolados e a testemunha) x 2 (dois métodos de aplicação) x 5 (diferentes profundidades). Os dados foram submetidos à análise de variância. As médias referentes aos diferentes métodos de aplicação foram comparadas entre si pelo teste Scott-Knott $(\mathrm{P}<0,05)$, enquanto que as médias referentes ao desempenho de cada tratamento, quanto ao deslocamento, foram estudadas por regressão $(\mathrm{P}<0,05)$.

\section{RESULTADOS E DISCUSSÃO}

\section{Deslocamento na coluna de areia}

Foi possível observar que ambos os isolados foram eficientes no deslocamento pelo método coluna de areia, causando mortalidade mínima de $72 \%$ na menor concentração (CCA) e máxima de $92 \%$ na maior concentração (CCA e JPM3). Não houve diferença entre os dois isolados em nenhuma das concentrações testadas (Tabela 1).

Stuart et al. (1997) desenvolveram trabalho semelhante, para avaliar a capacidade de deslocamento de várias espécies de nematóides, objetivando o controle de Dysmicoccus vacinni (Hemiptera: Pseudococcidae). Segundo os autores, $H$. bacteriophora e $H$. indicus causaram até $90 \%$ de mortalidade na concentração de 500 JIs/placa.

Também Poinar \& Hom (1986) avaliaram a capacidade de deslocamento de $S$. carpocapsae, em condições de laboratório. Segundo eles, fatores como temperatura, umidade e tipo de solo podem influenciar na capacidade de deslocamento dos JIs.
Vários fatores podem estar envolvidos no processo de deslocamento de JIs. Esses fatores podem ser ambientais como umidade, composição e granulometria do solo, temperatura, presença ou não de hospedeiro, liberação de substâncias de sinalização pelas plantas atacadas entre outros; e também próprios do nematóide como idade e reserva de energia (Poinar \& Hom, 1986; Tol et al., 2001; Wennemann et al., 2004; Rasmann et al., 2005; Fitters \& Griffin, 2006).

\section{Deslocamento em coluna de solo}

No experimento de deslocamento em coluna de solo, foram avaliados os fatores independentemente e também a interação entre os dois isolados avaliados com os métodos de aplicação e com a profundidade, e ainda a interação dos métodos de aplicação com a profundidade. A interação método de aplicação e profundidade, não foi significativa, entretanto, o fator isolado e o método de aplicação quando combinados com a profundidade, apresentaram interações significativas.

Verificou-se que o isolado JPM3 foi mais eficiente para a cochonilha $D$. texensis em ambos os métodos de aplicação, causando 84 e $60 \%$ de mortalidade, quando aplicado em suspensão aquosa e cadáver infectado, respectivamente. Já o isolado CCA demonstrou-se eficiente quando aplicado em suspensão aquosa, causando $68 \%$ de mortalidade, porém, quando aplicado na forma de cadáver infectado, causou apenas $15 \%$ de mortalidade (Tabela2).

Quanto à interação isolado x método de aplicação, o tratamento suspensão aquosa JPM3 apresentou diferença significativa, causando $84 \%$ de mortalidade. $\mathrm{O}$ isolado CCA também diferiu da testemunha, apresentando valor máximo de $68 \%$ de mortalidade (Tabela2).

No tratamento cadáver infectado, o resultado foi semelhante, porém, a porcentagem de mortalidade foi inferior ao tratamento suspensão aquosa, sendo os valores máximos de mortalidade de 15 e $60 \%$ para CCA e JPM3, respectivamente.

Comparando os dois métodos de aplicação do nematóide, pode-se observar que a aplicação em suspensão aquosa foi a mais eficiente para ambos os isolados, sendo o CCA e o JPM3 causaram um índice de mortalidade de aproximadamente 4,5 e 1,4 vezes, respectivamente, superior à mortalidade causada pelo nematóide aplicado por meio do método cadáver infectado.

Comparando os dois métodos de aplicação dentro de cada isolado, ambos apresentaram variação significativa. O isolado CCA causou $68 \%$ de mortalidade no tratamento suspensão aquosa e apenas $15 \%$ no tratamento cadáver infectado, enquanto que o isolado JPM3 causou $60 \%$ de mortalidade nos tratamentos cadáver infectado e $84 \%$ no tratamento suspensão aquosa, tornando evidente seu 
melhor desempenho em ambos os métodos de aplicação na busca de $D$. texensis.

A interação método $\mathrm{x}$ profundidade também foi significativa. Analisando primeiramente o fator métodos de aplicação, nas diferentes profundidades para cada isolado, o isolado CCA, aplicado por meio do método suspensão aquosa foi mais eficiente no deslocamento, causando 88 e $55 \%$ de mortalidade na menor e maior profundidade, respectivamente. Por outro lado, a aplicação do nematóide por meio do método cadáver infectado, apresentou baixa eficiência, causando na menor profundidade mortalidade máxima de $23 \%$ e, na maior profundidade causou mortalidade de apenas $5 \%$ (Fig. 1).

Em relação ao isolado JPM3, o método de aplicação do nematóide por meio de suspensão aquosa também foi mais eficiente, com o índice de mortalidade variando entre 90 e $68 \%$, entre a menor e maior profundidade. No tratamento cadáver infectado, o valor máximo de mortalidade foi obtido na profundidade de $10 \mathrm{~cm}(73 \%)$, e o menor, na profundidade de $20 \mathrm{~cm}$ (43\%) (Fig. 2).

Analisando os dois isolados avaliados, dentro de cada método de aplicação, podemos observar que, no método cadáver infectado, JPM3 apresentou melhores resultados que o isolado CCA (Fig. 3). Resultados semelhantes foram observados no método de suspensão aquosa (Fig. 4), com exceção na profundidade $5 \mathrm{~cm}$, onde o desempenho dos dois isolados foi semelhante. Porém, à medida que a profundidade foi aumentada, o isolado JPM3 causou maior mortalidade, quando comparado ao isolado CCA.

A produção de nematóides entomopatogênicos em larga escala tem evoluído nos últimos anos, bem como os métodos de estocagem desse tipo de produto. Entre as novas formas de estocagem, o uso de cadáveres infectados é uma forma promissora de aplicação de nematóides entomopatogênicos no controle de insetos-praga, apresentando, entre outras vantagens, a diminuição nos custos e a ausência do estresse físico, provocado no método de suspensão aquosa (Shapiro-Ilan et al., 2001).

No entanto, de acordo com os dados obtidos neste trabalho, a aplicação de nematóides pelo método de cadáver infectado teve desempenho inferior à aplicação via suspensão aquosa, para ambos os isolados avaliados. Uma possível explicação seria a diferença de umidade, já que no tratamento de cadáver infectado não foi aplicado água no momento da inoculação. Este fator pode ter influenciado na emergência e mesmo no deslocamento dos JIs, na coluna de solo.

Por outro lado, na ausência de condições favoráveis (umidade, temperatura e presença de outro hospedeiro), os JIs podem persistir dentro do hospedeiro, esperando por condições adequadas para sua emergência.

Quanto à dispersão vertical dos nematóides, vários fatores podem influenciá-la. Boff \& Smits (2001) estudaram a influência da densidade, idade e presença do hospedeiro na dispersão de $H$. megidis, Segundo os autores a dispersão é maior quando os JIs estão em altas densidades e quando são mais jovens.

Tabela 1 - Porcentagem de mortalidade de Dysmicoccus texensis em três diferentes concentrações de Heterorhabditis sp.(CCA e JPM3) aplicados sobre a coluna de areia.

\begin{tabular}{lccc}
\hline & \multicolumn{3}{c}{ Concentrações (JIs/inseto) ${ }^{1}$} \\
\cline { 2 - 4 } Isolados & 50 & 100 & 500 \\
\hline Heterorhabditis sp.CCA & $72 \pm 0,12 \mathrm{a}$ & $90 \pm 0,03 \mathrm{a}$ & $92 \pm 0,00 \mathrm{a}$ \\
Heterorhabditis sp. JPM3 & $76 \pm 0,04 \mathrm{a}$ & $92 \pm 0,00 \mathrm{a}$ & $92 \pm 0,00 \mathrm{a}$ \\
Testemunha & $4 \pm 0,00 \mathrm{~b}$ & $4 \pm 0,00 \mathrm{~b}$ & $4 \pm 0,00 \mathrm{~b}$ \\
\hline
\end{tabular}

${ }^{1}$ Médias seguidas por letras distintas minúsculas nas colunas diferem entre si pelo teste Scott-Knott $(\mathrm{P}<0,05)$.

C.V. $=8,20 \%$

Tabela 2 - Porcentagem média de mortalidade de Dysmicoccus texensis causada pelos isolados Heterorhabditis sp. CCA e Heterorhabditis sp. JPM3 em coluna de solo aplicados pelos métodos de suspensão aquosa e cadáver infectado.

\begin{tabular}{lcccc}
\hline \multirow{2}{*}{ Tratamentos } & \multicolumn{4}{c}{ Métodos de Aplicação } \\
\cline { 2 - 5 } & \multicolumn{3}{c}{ Suspensão Aquosa } & \multicolumn{2}{c}{ Cadáver Infectado } \\
\hline CCA & $68 \pm 13,00$ & $\mathrm{Ab}^{1}$ & $15 \pm 12,00$ & $\mathrm{Bb}$ \\
JPM3 & $84 \pm 12,20$ & $\mathrm{Aa}$ & $60 \pm 21,00$ & $\mathrm{Ba}$ \\
Testemunha & $7 \pm 8,00$ & $\mathrm{Ac}$ & $7 \pm 8,00$ & $\mathrm{Ac}$ \\
\hline
\end{tabular}

${ }^{1}$ Médias seguidas de letras iguais maiúsculas nas linhas e minúsculas nas colunas não diferem entre si pelo teste de médias ScottKnott $(\mathrm{P}<0,05)$. 


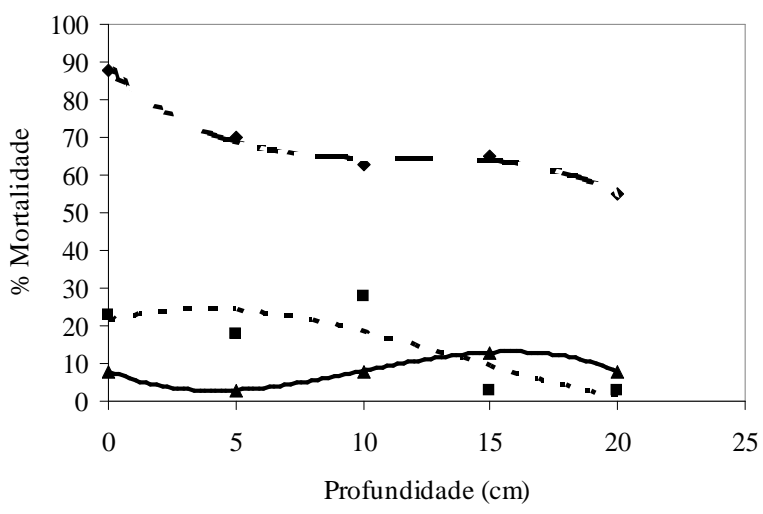

- Suspensão - Cadáver $\Delta$ Testemunha

Testemunha $=-0,0133 \mathrm{x}^{3}+0,4 \mathrm{x}^{2}-2,9667 \mathrm{x}+8$

Cadáver $=-0,0093 \mathrm{x}^{3}-0,417 \mathrm{x}^{2}+3,5095 \mathrm{x}+64,94$

$\mathrm{R}^{2}=1$

Suspensão $=-0,028 \mathrm{x}^{3}+0,7543 \mathrm{x}^{2}-4,9857+91,514$

$\mathrm{R}^{2}=0,99$

$\mathrm{R}^{2}=0,68$

Figura 1 -Curva de regressão para o isolado CCA, aplicado pelos métodos suspensão aquosa e cadáver infectado, considerando a porcentagem de mortalidade de Dysmicoccus texensis em função da profundidade em $\mathrm{cm}$.

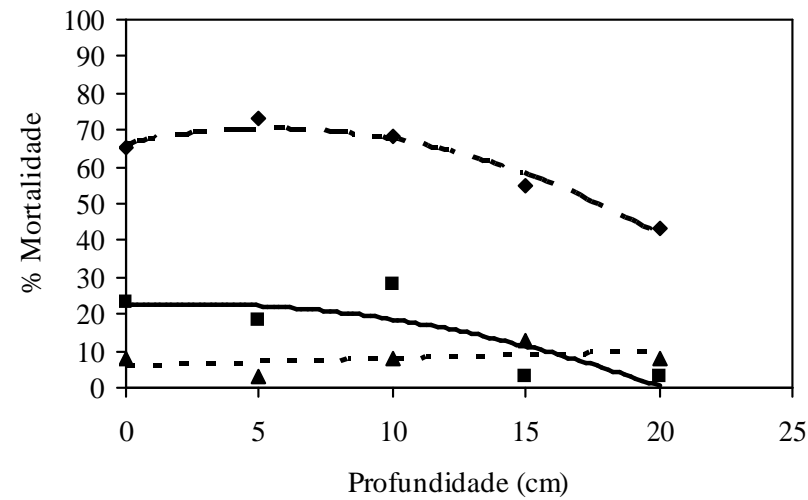

$\bullet$ JPM3 $\square$ CCA $\triangle$ Testemunha

JPM3 $=-0,1371 x^{2}+1,5029 x+66$ $\mathrm{CCA}=-0,0714 \mathrm{x}^{2}+0,3286+22,429$

$\mathrm{R}^{2}=0,66$

Testemunha $=-16 \mathrm{x}^{2}+0,2 \mathrm{x}+6$ $\mathrm{R}^{2}=0,2$

Figura 3 - Curva de regressão para o isolado JPM3, CCA e Testemunha, aplicados pelo método cadáver infectado, considerando a porcentagem de mortalidade de Dysmicoccus texensis em função da profundidade em $\mathrm{cm}$.

\section{CONCLUSÃO}

De maneira geral, o isolado JPM3 teve melhor desempenho que o isolado CCA, quanto à capacidade de busca pela cochonilha-da-raiz-do-cafeeiro. O método de aplicação por suspensão aquosa também

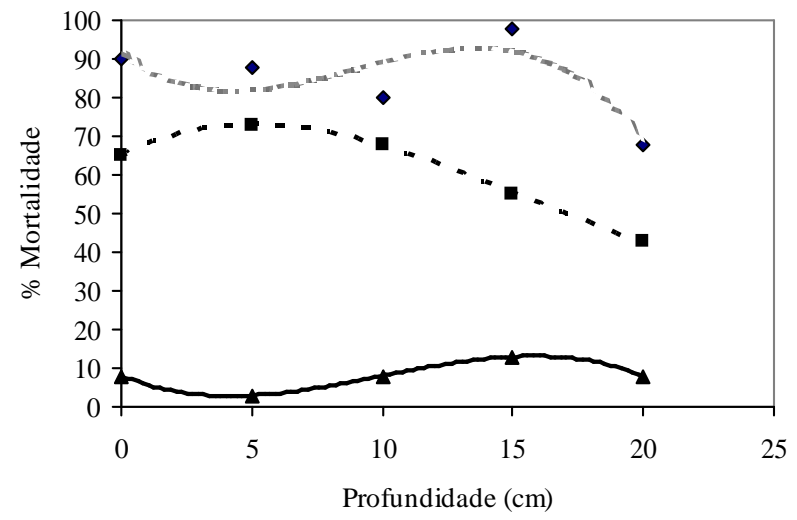

- Suspensão $\boldsymbol{- C a d a v e r ~} \Delta$ Testemunha

Testemunha $=-0,0133 \mathrm{x}^{3}+0,4 \mathrm{x}^{2}-3,9667 \mathrm{x}+8 \quad \mathrm{R}^{2}=1$

Cadáver $=-0,0153 \mathrm{x}^{3}+0,5314 \mathrm{x}^{2}-6,1452 \mathrm{x}+83,231 \mathrm{R}^{2}=0,99$

Suspensão $=-0,0067 \mathrm{x}^{3}-0,2714 \mathrm{x}^{2}-1,7619 \mathrm{x}+21,42 \mathrm{R}^{2}=0,67$

Figura 2 - Curva de regressão para o isolado JPM3, aplicado pelos métodos suspensão aquosa e cadáver infectado, considerando a porcentagem de mortalidade de Dysmicoccus texensis em função da profundidade em $\mathrm{cm}$.

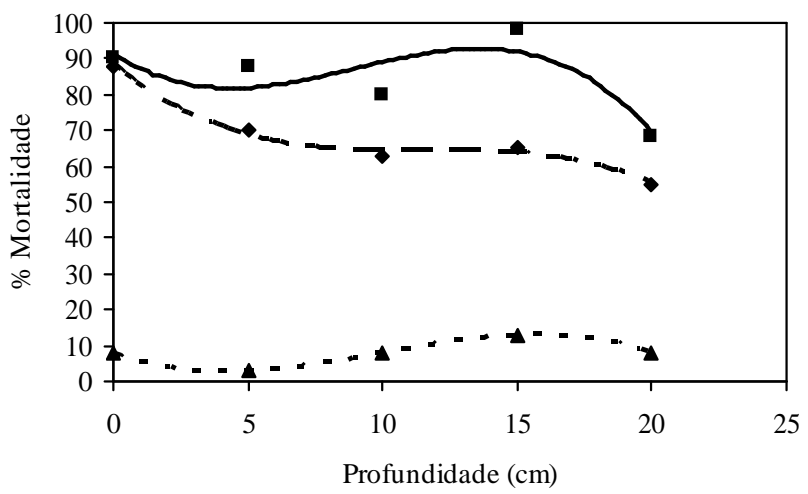

- CCA - JPM3 $\triangle$ Testemunha

JPM3 $=-0,028 x^{3}+0,7543 x^{2}-4,9857 x+91,514 \quad R^{2}=0,89$ $\mathrm{CCA}=-0,0153 \mathrm{x}^{3}+0,5314 \mathrm{x}^{2}-6,1452+86,231 \quad \mathrm{R}^{2}=0,99$ Testemunha $=-0,0133 x^{3}+0,4 x^{2}-2,6667 x+8 \quad R^{2}=1$

Figura 4 - Curva de regressão para o isolado JPM3, CCA e Testemunha, aplicados pelo método suspensão aquosa, considerando a porcentagem de mortalidade de Dysmicoccus texensis em função da profundidade em $\mathrm{cm}$.

se mostrou mais eficiente que o método de cadáver infectado. Além disso, a eficiência de ambos os isolados é inversamente proporcional à profundidade, ou seja, a eficiência diminui à medida que a profundidade aumenta. 


\section{REFERÊNCIAS BIBLIOGRÁFICAS}

ANDALÓ, V.; MOINO JÚNIOR, A.; SANTA-CECILIA, L.V.C.; SOUZA, G.C. Compatibilidade de Beauveria bassiana com agrotóxicos visando o controle da cochonilha-da-raiz-do-cafeeiro Dysmicoccus texensis (Tinsley) (Hemiptera: Pseudococcidae). Neotropical Entomology, Londrina, v.33, n.4, p.463-467, jul./ago. 2004a.

ANDALÓ, V.; MOINO JÚNIOR, A.; SANTA-CECILIA, L.V.C.; SOUZA, G.C. Seleção de isolados de fungos e nematóides entomopatogênicos pra a cochonilha-daraiz-do-cafeeiro Dysmicoccus texensis (Tinsley). Arquivos do Instituto Biológico, São Paulo, v.71, n.2, p.181-187, abr./jun. 2004b.

BOFF, M.I.C.; SMITS, P.H. Effects of density, age and host cues on the dispersal of Heterorhabditis megidis. Biocontrol Science Technology, San Diego, v.11, n.4, p.505-514, Aug. 2001.

BRIEN, W.J. o'; EVANS, B.I.; BROWMAN, H.I. Flexible search tactics and efficient foraging in saltatory searching animals. Oecologia, New York, v.80, n.1, p.100-110, 1989.

FITTERS, P.F.L.; GRIFFIN, C.T. Survival, starvation, and activity in Heterorhabditis megidis (Nematoda: Heterorhabditidae). Biological Control, San Diego, v.37, n.1, p.82-88, 2006.

GEORGIS, R.; KOPPENHOFER, A.M.; LACEY, L.A.; BÉLAIR, G.; DUNCAN, L.W.; GREWAL, P.S.; SAMISH, M.; TAN, L.; TOL, R.W.H.M. van. Successes and failures in the use of parasitic nematodes for pest control. Biological Control, San Diego, v.37, n.1, 2005.

HUEY, R.B.; PIANKA, E.R. Ecological consequences of foraging mode. Ecology, Washington, v.62, n.4, p.991-999, 1981.

KAYA, H.K. Entomopatogenous nematodes for insect control In IPM system. In: HASS, M.A.; HERZOG, D.C. Biological control in agricultural IPM systems. New York: Academic, 1985. p.283-302.

LEWIS, E.E.; CAMPBELL, J.; GRIFFIN, C.; KAYA, H.; PETERS, A. Behavioral ecology of entomopathogenic nematodes. Biological Control, San Diego, v.38, n.1, p.66-79, July 2006.

NAKANO, O. O estudo da cochonilha da raiz do cafeeiro, Dysmicoccus cryptus (Hempel, 1918) comb.n. (Homoptera: Pseudococcidae). 1972. 130p. Tese (Livre Docência)-Escola Superior de Agricultura Luiz de Queiroz, Piracicaba, 1972.
POINAR, G.O.; HOM, A. Survival and horizontal movement of infective stage Neoplectana carpocapsae in the field. Journal Nematology, Lakehand, v.18, n.1, p.34-36, Jan. 1986.

RASMANN, S.; KOLLNER, T.G.; DEGENHARDT, J.; HILTPOLD, I.; TOEPFER, S.; KUHLMANN, U.; GERSHENZON, J.; TURLINGS, T.D.J. Recruitment of entomopathogenic nematodes by insect-damaged maize roots. Nature, London, v.434, n.7034, p.732-737, Abr. 2005.

SALGADO, S. M. L.; RESENDE, M. L. V.; CAMPOS, V. P. Efeito de indutores de resistência sobre Meloidogyne exigua do cafeeiro. Ciência e Agrotecnologia, v.31, n.4, p.1007-1013, jul./ago., 2007.

SANTA-CECILIA, L.V.C.; SOUSA, J.C.; REIS, P.R. Novas constatações da cochonilha-da-raiz Dysmicoccus criptus em lavouras de café no sul de Minas Gerais. Lavras: Epamig, 2000. 2p. (Epamig. Circular técnica, 130).

SHAPIRO-ILAN, D.I.; LEWIS, E.E.; BEHLE, R.W.; McGUIRES, M.R. Formulation of entomopathogenic nematode-infected cadavers. Journal of Invertebrate Pathology, San Diego, v.78, n.1, p.17-23, July 2001.

SOUZA, J.C.; REIS, P.R.; SANTA-CECILIA, L.V.C.; DAUM, S.; SOUZA, M. de A. Cochonilha-da-raiz do cafeeiro: aspectos biológicos, dano e controle. Lavras: Epamig, 2001. 4p. (Epamig. Circular técnica, 136).

SOUZA, J.C.; RIBEIRO, J.A. Cochonilha-da-raiz: cafeicultor, conheça e saiba como controlar esta praga com inseticidas neonicotinóides. Lavras: Epamig, 2003. 3p. (Epamig. Circular técnica, 162).

STUART, R.J.; POLAVARAPU, E.; LEWIS, E.; GAUGLER, R. Differential susceptibility of Dysmicoccus vacinni (Homoptera: Pseudococcidae) to entomopathogenic nematodes (Rhabditida: Heterorhabditidae and Steinernematidae). Journal of Economic Entomology, Lanham, v.90, n.4, p.925-932, Aug. 1997.

TOL, R.W.H.M. van; SOMMEN, A.T.C.M. van; BOFF, I.C.; BEZOOIJEN, J. van; SABELIS, M.W.; SMITS, P.H. Plants protect their roots by alerting the enemies of grubs. Ecology Letters, Oxford, v.4, n.4, p.292-294, July 2001.

WENNEMANN, L.; SHANKS, C.H.; SMITH, K.A.

Movement of entomopathogenic nematodes in soils of Fragaria spp. Commun Agricultural Applied Biological Science, Ghent, v.69, p.347-357, 2004. 